\title{
Computer games for user engagement in Attention Deficit Hyperactivity Disorder (ADHD) monitoring and therapy
}

\author{
Michael P. Craven ${ }^{1,2}$, Madeleine J. Groom ${ }^{1,3}$ \\ ${ }^{1}$ NIHR MindTech Healthcare Technology Cooperative \\ Institute of Mental Health, \\ University of Nottingham Innovation Park, Triumph Road \\ Nottingham, NG7 2TU. United Kingdom
}

\author{
${ }^{2}$ The University of Nottingham, Faculty of Engineering \\ Electrical Systems \& Optics Research Division \\ michael.craven@nottingham.ac.uk \\ ${ }^{3}$ The University of Nottingham, Institute of Mental Health, \\ Division of Psychiatry and Applied Psychology \\ maddie.groom@nottingham.ac.uk
}

\begin{abstract}
State-of-the-art computer games and psychological tests for symptom monitoring and therapy in Attention Deficit Hyperactivity Disorder (ADHD) are explored and reviewed. Three foci for research studies are identified: task (human performance) focus; educational focus; medical/clinical focus.

It is found that game designs in the literature include a variety of tests of cognition mostly dependent on attention and executive functions (inhibitory motor control, working memory, interference suppression) which involve reactions to stimuli on computer (or mobile phone) screens. In addition, based on the measurement of neural pathways that can be accessed by Brain Computer Interfaces, there are several applications of games that employ biofeedback and demand the user to control aspects of their brain activity to play them, with the aim of improving function. A number of games have been used in clinical studies for self-monitoring and therapy, some of these controlled with comparators such as treatment as usual or cognitive therapies, or with the individual as their own control, where efficacy is evaluated by measuring behavioural and functional outcomes on measurement instruments such as ADHD or behavioural trait questionnaires or other cognitive tests. Other applications of games include education and raising awareness of mental health conditions to reduce stigma.

The paper then presents and proposes designs of new games that are based on psychological tests or tasks that aim to monitor or improve attention, inhibitory and/or motor activity including Continuous Performance Tests, Go/No-go and Stop-signal tasks.
\end{abstract}

Keywords-healthcare, gamification, e-Health, m-Health, pervasive and ubiquitous computing

\section{INTRODUCTION}

\section{A. 'Gamification' of health}

Games are an attractive means of engaging people in medical interventions and clinical studies and to encourage healthy behaviours or increase health awareness and education [1-3]. Computer games and simulations are also the subject of investigations into aspects of human performance and the effect of play on improvement [4]. For the purpose of medical intervention, computer games are a way of discovering or monitoring the symptoms of a specific disease by measuring function in a controlled and engaging way. The ubiquity of mobile platforms and wearable technology in recent years supports the individual in conducting less burdensome and more regular (even continuous) self-monitoring that could contribute to better health outcomes. For mental health in particular, games can be aimed at not only monitoring but also improving function for a person with a specific diagnosis or symptom, or at reducing the severity of negative behavioural traits that at their extremes are associated with a range of disorders.

\section{B. $A D H D$}

Attention Deficit Hyperactivity Disorder (ADHD, AD/HD or $\mathrm{ADD}$ ) is a common neurodevelopmental condition that starts in childhood and, for the majority of individuals, persists into adult life [5]. The symptoms of ADHD can include: inattention (difficulty concentrating for long or finishing tasks, disorganised, easily distracted, forgetful, unable to listen when people are talking), hyperactivity (fidgety, unable to sit still, talking excessively, always on the go, feeling of inner restlessness), and impulsivity (acting quickly without thinking about the consequences, interrupting other people, difficulty waiting turn). As well as having a distinct diagnosis, ADHD differs from the normal spectrum of a human population because there are levels of these behaviours that result in significant psychological, social and/or educational or occupational impairment that occurs across multiple domains and settings and persists over time [6].

Whereas the most widely accepted method of assessment of ADHD is the clinician's judgement, which may employ a range of questionnaires based on parent and/or teacher observations as well as the individual's and clinician's, objective methods based on psychometric and cognitive tests are now commonly available in the assessment of ADHD in children and adults. One such example is the QbTest desktop PC system that uses a game-like continuous performance test (CPT) and tracks head motion during the test [7]. The results of tests including errors and amount of motion can be presented numerically or graphically to patients and used to show change after drug treatment.

Post-diagnosis, monitoring in ADHD is currently aimed predominantly at detecting non-response or adverse response to medication, the main treatment for ADHD. Monitoring is particularly important in the early 'titration' stage where the therapeutic dose is being found. There is also an emphasis on ensuring good medication adherence and this may provide a role for games through motivational action. 
Furthermore, based on National Institute for Health and Care Excellence (NICE) guidelines in the UK [6], treatment for ADHD may include psychological treatment such as group or individual cognitive behavioural therapy (CBT) and/or social skills training, its use being dependant on age and severity and whether parenting-training/education or drug treatments have been effective.

Therapies or monitoring of clinical interventions using games are not in common practice but are of considerable research interest, as outlined in the next section.

\section{REVIEW}

\section{A. General}

The source of literature relevant to the study of games in ADHD is quite diverse. This is a result of different foci which may be considered to fall into three main areas (illustrated in Fig. 1.): a task (human performance) focus; an educational focus; and a medical focus. In all cases the goal may be either to inform or improve, or both. A task focussed study may remain in the context of the specific game or games that require similar abilities, aiming to better understand the perceptual or cognitive processes contributing to performance on these and how performance may be enhanced (a near transfer effect), whereas an educational focus may be more interested in a particular learned ability such as reading speed, for example, where a transfer from the context of game playing to improved reading is the aim (far transfer).

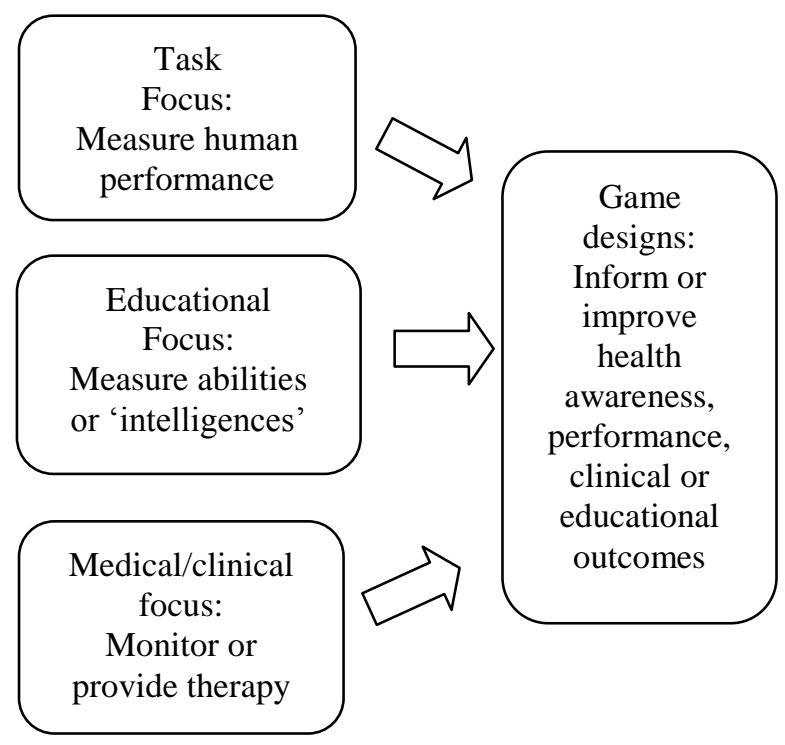

Fig. 1. Approaches to games for ADHD with three foci: task (human performance), educational, and medical/clinical monitoring or therapeutic intervention, to inform or improve outcomes

For a medical/clinical focus the goal of the game may be to monitor or, in the case of therapy, to affect a particular cognitive function or trait and measure outcomes on an ADHD-specific clinical scale or questionnaire about the individual's behaviour, hopefully reflecting a real world improvement in quality of life from the intervention, whether this is achieved directly from the game playing or from another treatment (e.g. drug, CBT).
As a result of this diversity, terminology about therapy is also diverse and includes psychotherapy, cyber-therapy or play therapy, or simply treatment or intervention. Another categorisation concerns the interface which may either require fine motor control of hands or eye-gaze with respect to visual or auditory stimuli, or else be based on neural pathways that can be accessed by Brain Computer Interfaces which activity recording techniques such as Electroencephalography (EEG) [8]. Other modes of biofeedback draw on measures of heartrate variability (linked to breathing control) and skin conductance [9].

\section{B. Evidence}

Using the previous three foci, examples from the literature of games and ADHD or related cognitive tests can be divided as follows:

Task focus

- Abilities of game players (action video games, puzzle games) [4, 10-13]

Education/Training/Health Awareness focus

- Cyber-therapy classroom [14]

- Intelligences [15]

- Health awareness [16, 17]

- $\quad$ Cognitive (working memory) training [18]

Medical/clinical focus

- $\quad$ CBT (cognitive behavioural therapy) [19]

- Cognitive tests: CPT (continuous performance test) [7, 20, 21]; Stop-signal or Go/No-go [22, 23]

- Working memory [24-27]

- $\quad$ Eye-gaze [28-30]

- Neurofeedback/Brain Computer Interfaces: EEG (electroencephalogram) theta/beta $[31,32]$; SCP (slow cortical potentials) $[33,34]$

- Other biofeedback [9]

It can be seen that most examples of games in the above list are concerned either with tasks to assess the effect of game playing on human performance or are for therapy, and a number of these are based on cognitive tests used more generally. One CPT game devised by the authors and other colleagues, SnappyApp, was intended for monitoring only [21] (see later), while another, QbTest, has been developed to aid diagnosis and monitoring [7].

\section{Task focus}

From the literature focussing on human performance, attention or perception tests have been used to compare video game players versus non-players [4]. These have included flanker compatibility (spotting specified shapes in the visual field whilst requiring inhibition from distractor shapes in the periphery) aimed at measuring processing speed as the task is made more difficult, enumeration (counting squares presented 
momentarily and measuring accuracy as the number is increased), localisation accuracy of stimuli objects over space (circles on spokes at different eccentricities) and a blink test (detection of a specified letter stimulus after a task-relevant target stimulus) [4]. Oei and Patterson have investigated executive function of players who were asked to play a variety of commercial action and puzzle games, administering a battery of tests including random task-switching, flanker and response inhibition (Go/No-go) [12, 13]. Memory related tests used to assess the ability of video games players include Posner letter identity, proactive inference, $N$-back and visual short-term memory [10].

Interestingly, these studies have tended to show enhanced cognitive function in habitual gamers compared with nongamers and, by exposing non-gamers to intensive videogaming sessions, have shown that these effects may be attributable to the video games themselves, rather than individual differences in specific cognitive features that predispose some people to play video games. Whether or not these benefits are also found in people with neurodevelopmental disorders has not been investigated, but there are likely to be issues around feasibility and acceptability to parents and clinicians of attempting to use these in any therapeutic capacity in ADHD.

\section{Educational or medical/clinical focus}

Considering the educational and/or clinically therapeutic literature, the aim (as discussed earlier) has been to develop and evaluate game-based systems that can enhance some aspect of function, thought to be central to the impairment in question. Games that have been developed specifically with a therapeutic aim for ADHD have often been coupled with neurofeedback, based on evidence that neurofeedback may be an efficacious and safe treatment for ADHD [35]. Neurofeedback (Brain Computer Interface) games described in the literature are based either on control of the ratio between slow (theta, 4-7Hz) and fast (beta, $13-20 \mathrm{~Hz}$ ) oscillatory brain waves (theta/beta ratio) measured in the electroencephalogram (EEG) [31], or the modulation of slow cortical potentials (SCP) amplitude, an index of low frequency large amplitude brain waves related to arousal regulation [33, 34].

Participants are taught to self-regulate these features of the EEG by focussing their attention on specific aspects of the game in question. For instance, theta/beta ratio was mapped to horizontal or vertical control of game objects such as the diver/dolphin in Play Attention that is required to find treasure at the bottom of the sea whilst avoiding predators [31]. Alternatively, SCP amplitude was modulated by players focussing on raising a dot or arrow as it traversed the screen from left to right with feedback every 0.5 seconds [33]. Another involved a pole-vaulting mouse, the player being required to change the colour of the pole according to the SCP amplitude in relation to its baseline, to enable the mouse to jump [34]. An alternative neurofeedback approach reported recently (in the Cogo Land game) is to identify individualised EEG features reflecting attention and train the ability to modulate this parameter [32].

At the heart of these neurofeedback methods is the principle that improved self-regulation of particular brain states transfers to better function on other outcomes, including cognition and symptoms or behaviours in ADHD [35]. What is less clear is precisely how these altered brain states, such as lower theta/beta ratio, elicit this improved function, or in the case of Cogo Land where an individualised EEG parameter is identified, how this relates to other more widely reported EEG indices. Perhaps this is not important provided the therapeutic effect is reliable, but the evidence attesting to neurofeedback is undermined by a lack of double-blind randomised controlled trials [36]. Moreover, others have argued that it is preferable to develop therapeutic interventions in ADHD for which the mechanisms of action are well understood. Gaze control is one example of this.

Gaze control games require fixation of gaze on moving game objects (such as in the Circus and Bubble games of Tarkeezy/Attentive Eye [28]) or the switching of gaze to different parts of the screen (Attentive Eye Matrix game), or demand response inhibition of distraction objects in the visual periphery such as in RECOGNeyes [29]. The basis of these games is that the oculomotor system is intimately linked to the attention and inhibitory motor control systems. Thus, enhancing gaze control should lead to improvements in other non-trained cognitive functions and ADHD symptoms, on the assumption that impairments in attention and inhibitory control are core to ADHD.

Another example of the development of a therapy based on a clear hypothesis about a specific cognitive target is CogMed developed by Klingberg et al. [24, 26, 27]. In this suite of computerised games, participants are presented with a range of working memory tasks, each designed to challenge and scaffold increments in working memory capacity. Together with evidence of alterations of neural markers in healthy adults [37], Klingberg et al. have had some success in improving parent ratings of ADHD symptoms [24, 26], although the transfer to other cognitive functions remains to be firmly established. This intervention has also been applied to improve working memory in children without a medical diagnosis [18], representing the capacity to use these games to improve function in children with a range of difficulties, rather than just those with a narrowly defined medical diagnosis.

\section{Previous work - Continuous Performance Test}

An example of a game associated with the third focus of this review, namely monitoring of symptoms over time, is our ongoing work on SnappyApp which is a Continuous Performance Test (CPT) running on a smartphone platform [21]. The original SnappyApp replicated an existing CPT-AX visual task [38] where letters of the alphabet are presented momentarily at central fixation on a computer screen and the player is required to respond only to presentations of the letter $\mathrm{X}$ immediately following an A (the $\mathrm{X}$ in such an AX sequence appearing $10 \%$ of the time during the entire 11 minute test) amongst a set of other letters presented in sequence (see Fig. 2).

For each CPT session, errors of omission (missed response to a target stimulus) and commission (response to non-target stimulus) and hand/body motion (via acceleration and rotation sensors of the mobile device) were recorded and analysed within test at each quarterly time period and then between 
multiple tests, for each subject. The test included features similar to the desktop-based ADHD assessment tool QbTest [7], which also monitors the three symptoms of inattention, impulsivity and hyperactivity, but with a mobile element to enhance the capacity for remote self-monitoring outside the clinic.

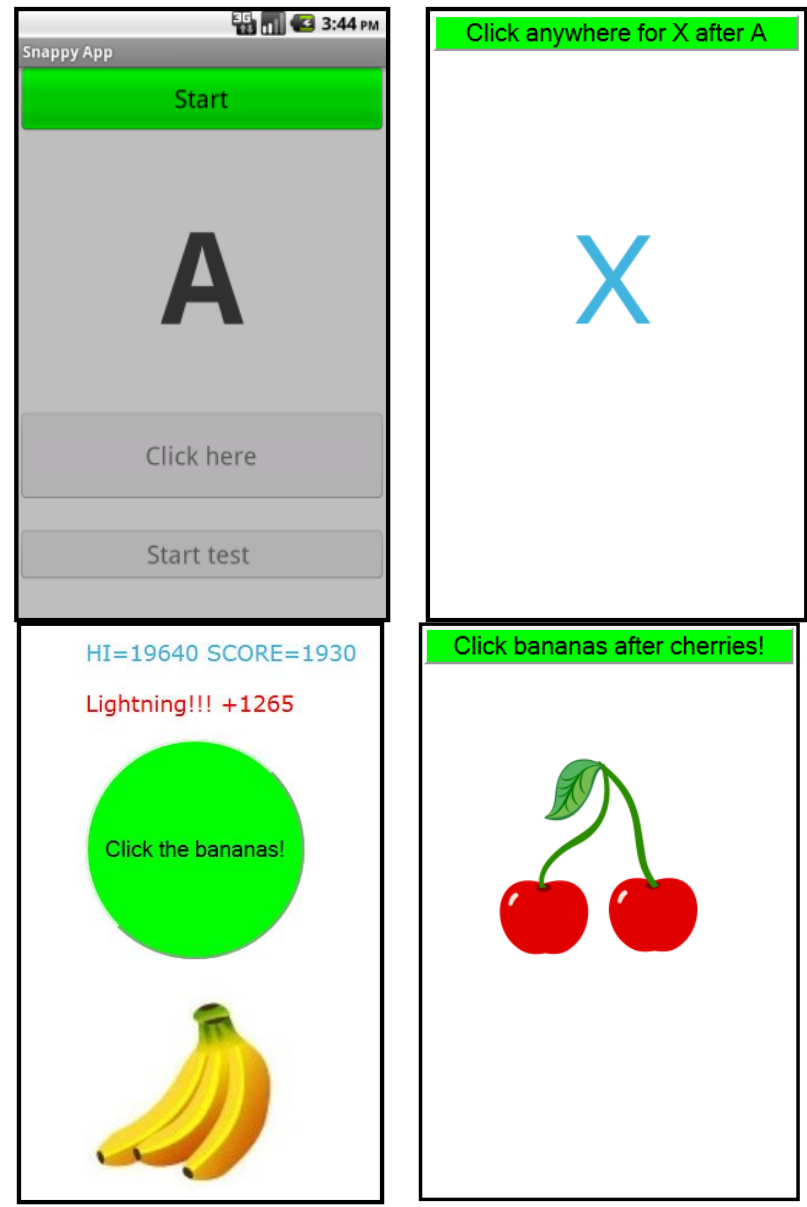

Fig. 2. SnappyApp Android and web-app CPT-AX designs, a prototype gamified CPT-X web-app version, and a moderately gamified CPT-AX

An initial CPT-AX study with adults was conducted, who completed an impulsivity questionnaire at each session. Another simpler variant of the test (CPT-X), suited to young children, was gamified to use fruit instead of letters, with bananas replacing the $\mathrm{X}$ stimulus, and also introduced scores. The CPT-X with bananas was subsequently adapted for a public awareness raising application Attention Grabber on deployed on large touch screens in urban areas [17], representing another potential output for games in ADHD. Later, some of the gaming elements were removed to avoid some of the distraction and a test analogous to CPT-AX, with bananas as the stimuli to be selected after cherries, was used in a school study with young people, as seen in the last image of Fig. 2. The score was available at the end of the test. Here, subjects completed an ADHD-specific questionnaire, the Strengths \& Weaknesses of ADHD (SWAN) [39].

In studies with these variants of CPT apps there was good test-retest reliability, deterioration in performance over the period of a test session for some dependent variables, and some significant correlations with traits in the general population which could be associated with ADHD (e.g. positive urgency in the adult study; correlation of omission errors with SWAN score in the young person study), however for other variables such as reaction time (RT) there was no significant correlation with ADHD traits and no deterioration over session time. Further work is needed to identify the key features of cognition that are useful for monitoring ADHD symptoms.

\section{NEW GAMES}

\section{A. Rationale}

We are the in process of extending the existing work with SnappyApp to produce new games for monitoring ADHD related behaviours and also to extend the application towards therapy, where appropriate.

Firstly, based on evidence that intra-individual variation in RT across a range of cognitive tests is a reliable marker of ADHD, we are currently developing a revised version of the game which will permit more accurate measurement of RT variability than allowed by the CPT with its focus on rare responses. To this end, we have drawn on the literature concerning Go/No-go and Stop-signal tasks which have relevance for ADHD. These tests rely on regular responses to stimuli presented, unless a No-go or Stop signal is shown [22, 23]. In the case of $\mathrm{Go} / \mathrm{No}$-go the appropriate response is required to each stimulus (respond as fast as possible to Go, null response for No-go) and in the case of Stop signals, a null response is required at a variable time after a Go stimulus. These tests therefore provide reliable measures of attention (target detection, RT variability) and impulsivity (inhibition of a rapid, automated response on No-go or Stop trials), which are two of the cardinal features of ADHD.

Secondly, in our current work we wish to draw on additional game design elements such as levels of difficulty to challenge regular users and so maintain motivation to monitor or to engage them in therapy. A wealth of research, beyond the scope of the present review, has identified impaired motivation and reduced sensitivity to rewards, particularly when these are delayed or infrequent as key factors in the cognitive and behavioural features of ADHD [40]. Overcoming these features of ADHD, to ensure adherence to and engagement in games that are designed to monitor or treat symptoms, is one of the most prominent challenges to research in this field.

We now present new game ideas that incorporate the key features of Go/No-go and Stop-signal tasks.

\section{B. Game designs and concepts}

Themes for Stop-signal or Go/No-go games can include cartoon-like characters.

We have designed a Go/No-go task for young people where the Go and No-go signals are cartoon graphics, where Go or No-go signal stimuli are presented momentarily in a random binary sequence and the player is asked to respond to Go and ignore No-go. The design for a newly implemented game, Awkward Owls, is shown in Fig. 3. 

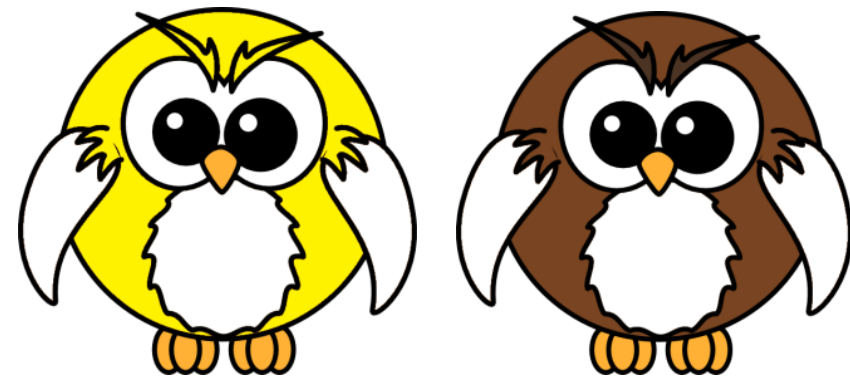

Fig. 3. Awkward Owls Go / No-go task graphics including yellow owl Go signal \& brown owl No-go signal. [Graphics adapted from openclipart.com]

In the game, the player is presented with a narrative where they are a conservation volunteer who is asked to collect rare yellow owls for a wildlife survey. They must collect only yellow owls and ignore the more well-known brown owls. As is usual with Go/No-go tests, brief and variable inter-stimulus intervals are used to make it harder to anticipate the upcoming stimulus, and so the player's response has to be kept primed and ready throughout. Scoring is based on reaction times to the Go and No Go stimuli, together with penalties for the number of missed Go selections and any incorrect No-go selections. The Go:No-go ratio is also recorded. After each response (if any), a message is displayed, either Hoot! or Boo!!! depending on the stimulus.

One issue in designing games based on cartoon characters (and especially if they are familiar ones) is that they need to be authentic but also that stimuli are easily decoded and are not biased (e.g. by size or shape difference) from a cognitive psychology perspective. Design choices therefore need to be made to ensure that these design constraints are addressed. For example, Go/No-go tasks are usually more difficult the greater the visual similarity between Go and No-go stimuli because it is then harder to differentiate between them in time to withhold the prepotent Go response. This is particularly so if global, low frequency visual features like shape are held constant (like the overall outline cast by the cartoon character), but less so with finer details, including differences in colour. In the Awkward Owls example this means changing the body of the No-go signal from brown to yellow to create the Go signal, but not to add too much of a difference in expression or other shape change. Whether these modifications are acceptable to players who are familiar with popular characters or expectations of expressive cues will need to be investigated before finalising the game design. On the other hand, the introduction of more gradual changes, such as colour transitions or to sizes of facial features, may be one possible way of increasing longer-term engagement in the game where greater similarity of stimuli is more challenging in terms of game play. So in the above example, to make the game more difficult at higher levels, the Go character may be only in transition to its yellow colour.

The Awkward Owls game based on the above design has been completed as a Javascript web-app to be deployed on a smartphone for data collection and analysis with the involvement of young people in public events such as Summer Scientist Week run by the School of Psychology, University of Nottingham. User experience will be captured to refine the game e.g. difficulty and length of the game and to explore level design.

Wormy Fruit is another potential game, this time based on a Stop-signal task, which like the SnappyApp CPT consists of a sequence of fruit being presented, with the fruit stimulus being the Go signal (persistent rather than momentary), however in this case there is a possibility of a fruit having a worm in it, and the presentation of a fruit with a worm in (at variable time after the Go signal) is the Stop-signal (Fig. 4).

Again, choices need to be made about the precise stimuli designs so that the Stop signal is readily decoded whilst maintaining a degree of authenticity. For example, it may be that a wormy apple that maintains similar size to the nonwormy apple is preferable, for the reason already explained in the Awkward Owls example.
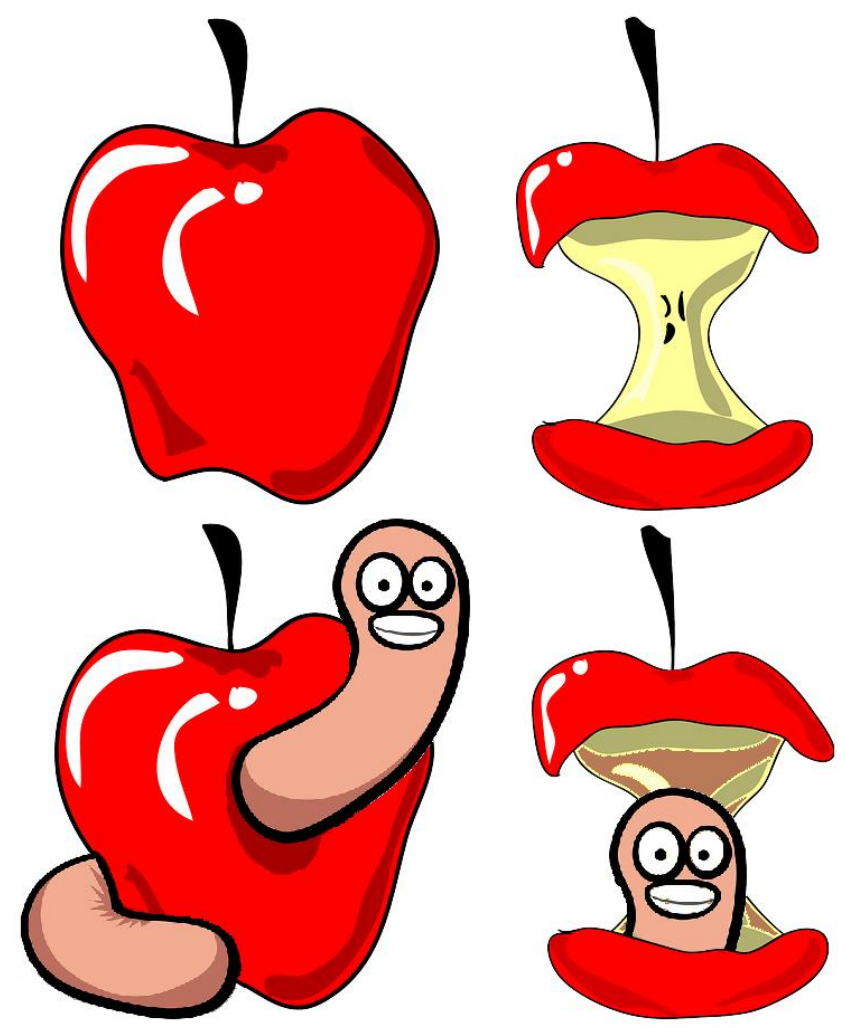

Fig. 4. Wormy Fruit concepts including Apple stimulus (Go signal), Eaten apple (Result of response to stimulus), Wormy apple (Stop signal), Eaten wormy apple (Result of missing Stop, including clicking early before Stop, when Apple is wormy). [Graphics used/adapted from 4vector.com and openclipart.com]

In Wormy Fruit, scoring can be based on some or all of:

- Eating fruit by clicking on them after the Go signal, possibly with different scores for different fruit

- Higher score for quicker reaction time (RT) on nonwormy fruit

- $\quad$ Penalty for waiting too long after Go on non-wormy fruit 
- Large score for waiting for what turns out to be a wormy apple, and ignoring it

- $\quad$ Penalty for clicking after Go but before Stop on what turns out to be wormy fruit, which outweighs fast RT

- $\quad$ Larger penalty clicking after Stop

The relative size of the scores will depend on the ratio of Go to Stop.

Level design ideas include:

- New fruit in each level with increasingly higher scores

- Modified presentation periods in later levels

- Different incidence ratio of Go to Stop stimulus incidence

- More bites to finish the fruit (multiple clicks) in higher levels

- Worm may appear only after more than one bite

Another game idea could involve diseases and antidotes where the player is asked to apply an antidote to change a character's colour.

\section{CONCLUSIONS}

This paper has provided a brief review and overview of the different foci for the use of games for ADHD or its related behaviours: a task (human performance) focus; an educational focus; and a medical/clinical focus.

One outcome of conducting the review is the observation that there is currently no game ontology [41] which includes cognitive or executive functions specifically for game description or design. This observation may be extended from ADHD to mental health applications of games in general, and could also incorporate aspects of physical ability. As such we hope to have contributed a step in rectifying this situation.

We have presented new game designs and concepts based on Go/No-go and Stop-signal tasks which are suited to monitoring in ADHD and also have potential for training of inhibitory control. This adds to work being carried out with gaze interfaces, some of which are aimed at training gaze control.

Additional game elements and mechanics should be considered for future developments. We note that Carr and Blanchfield have previously incorporated other game mechanics that are applied to engagement in mental health application in relation to their work with therapeutic virtual environments with children (including exploration, in-game communication, trade, character customisation, item collection and storyline) [42].

Another point for discussion is the level of evidence for therapeutic games in particular. Several of the citations include controlled clinical studies whereas others are more descriptive. A systematic review would be useful to assess this evidence more fully.

\section{ACKNOWLEDGEMENTS}

The research reported in this paper was conducted though the NIHR MindTech Healthcare Technology Co-operative (www.mindtech.org.uk - full sponsor link on first page). The authors wish to acknowledge Owen Williams who conducted the most recent CPT-AX fruit school study for his BMedPsych undergraduate project, his co-supervisor Prof. David Daley, and all of the SnappyApp study participants.

\section{REFERENCES}

[1] D. H. Goh, R. P. Ang, and H. C. Tan, "Strategies for designing effective psychotherapeutic gaming interventions for children and adolescents," Computers in Human Behavior, vol. 24, pp. 2217-2235, Sep 2008.

[2] L. Messineo, M. Allegra, and N. Alessi, "Game-Based Learning for Education and Training in the Health Sector," 2011 4th International Conference of Education, Research and Innovation (Iceri), pp. 3341-3353, 2011.

[3] M. P. Craven and L. Simons, "Games to engage: increasing mental health awareness," The Ergonomist, pp. 4-5, 2015.

[4] C. S. Green and D. Bavelier, "Action video game modifies visual selective attention," Nature, vol. 423, pp. 534-537, May 292003.

[5] R. A. Barkley, "International Consensus Statement on ADHD," J Am Acad Child Adolesc Psychiatry, vol. 41, p. 1389, Dec 2002.

[6] NICE. (2008, 09/06/2015). Attention deficit hyperactivity disorder: Diagnosis and management of ADHD in children, young people and adults. NICE guidelines [CG72]. Available: www.nice.org.uk/guidance/CG72

[7] QbTech. (2015, 09/06/2015). Identify, Rule Out and Monitor ADHD More Effectively With QbTest. Available: www.qbtech.com/qbtest.html

[8] T. S. Moriyama, G. Polanczyk, A. Caye, T. Banaschewski, D. Brandeis, and L. A. Rohde, "Evidence-Based Information on the Clinical Use of Neurofeedback for ADHD," Neurotherapeutics, vol. 9, pp. 588-598, Jul 2012.

[9] K. L. Amon and C. A. J., "Game-Based Learning: Current Research in Games for Health, a Focus on Biofeedback Video Games as Treatment for AD/HD," in Handbook of Research on Improving Learning and Motivation through Educational Games: Multidisciplinary Approaches, P. Felicia, Ed., ed Hershey, PA, USA: IGI Global, 2011, pp. 583-605.

[10] A. F. McDermott, D. Bavelier, and C. S. Green, "Memory abilities in action video game players," Computers in Human Behavior, vol. 34, pp. 69-78, May 2014.

[11] W. R. Boot, A. F. Kramer, D. J. Simons, M. Fabiani, and G. Gratton, "The effects of video game playing on attention, memory, and executive control," Acta Psychologica, vol. 129, pp. 387-398, Nov 2008.

[12] A. C. Oei and M. D. Patterson, "Enhancing Cognition with Video Games: A Multiple Game Training Study," Plos One, vol. 8, Mar 132013.

[13] A. C. Oei and M. D. Patterson, "Playing a puzzle video game with changing requirements improves executive functions," Computers in Human Behavior, vol. 37, pp. 216-228, Aug 2014.

[14] T. D. Parsons, T. Bowerly, J. G. Buckwalter, and A. A. Rizzo, "A controlled clinical comparison of attention performance in children with ADHD in a virtual reality classroom compared to standard neuropsychological methods," Child Neuropsychology, vol. 13, pp. 363-381, Jul 2007. 
[15] P. L. Baniqued, H. Lee, M. W. Voss, C. Basak, J. D. Cosman, S. DeSouza, et al., "Selling points: What cognitive abilities are tapped by casual video games?," Acta Psychologica, vol. 142, pp. 74-86, Jan 2013.

[16] T. A. Goldman, F. J. Lee, and J. Zhu, "Using video games to facilitate understanding of attention deficit hyperactivity disorder: a feasibility study," presented at the Proceedings of the first ACM SIGCHI annual symposium on Computerhuman interaction in play, Toronto, Ontario, Canada, 2014.

[17] M. P. Craven, Z. Young, L. Simons, H. Schnadelbach, and A. Gillott, "From Snappy App to Screens in the Wild: Gamifying an Attention Deficit Hyperactivity Disorder Continuous Performance Test for Public Engagement and Awareness," in 2014 International Conference on Interactive Technologies and Games (iTAG), Nottingham, United Kingdom, 16-17 October, 2014, pp. 36-43.

[18] D. E. Astle, J. J. Barnes, K. Baker, G. L. Colclough, and M. W. Woolrich, "Cognitive training enhances intrinsic brain connectivity in childhood," J Neurosci, vol. 35, pp. 6277-83, Apr 222015.

[19] H. Alshanqiti and P. Blanchfield, "Parameters for Video Games for the Treatment of Mental Illness in Children," Proceedings of the 3rd European Conference on Games Based Learning, pp. 1-7, 2009.

[20] F. Benedetti, N. Catenacci Volpi, L. Parisi, and G. Sartori, "Attention Training with an Easy-to-Use Brain Computer Interface," in Virtual, Augmented and Mixed Reality. Applications of Virtual and Augmented Reality. vol. 8526, R. Shumaker and S. Lackey, Eds., ed: Springer International Publishing, 2014, pp. 236-247.

[21] Z. Young, M. Craven, M. Groom, and J. Crowe, "Snappy App: A Mobile Continuous Performance Test with Physical Activity Measurement for Assessing Attention Deficit Hyperactivity Disorder," in Human-Computer Interaction. Applications and Services. vol. 8512, M. Kurosu, Ed., ed: Springer International Publishing, 2014, pp. 363-373.

[22] S. J. Johnstone, A. Dimoska, J. L. Smith, R. J. Barry, C. B. Pleffer, D. Chiswick, et al., "The development of stop-signal and Go/Nogo response inhibition in children aged 7-12 years: Performance and event-related potential indices," International Journal of Psychophysiology, vol. 63, pp. 25-38, Jan 2007.

[23] E. B. Liddle, G. Scerif, C. P. Hollis, M. J. Batty, M. J. Groom, M. Liotti, et al., "Looking before you leap: A theory of motivated control of action," Cognition, vol. 112, pp. 141-158, Jul 2009.

[24] T. Klingberg, "Computerized training of working memory in children with ADHD," European Neuropsychopharmacology, vol. 17, pp. S192-S193, Oct 2007.

[25] A. Chacko, A. C. Bedard, D. J. Marks, N. Feirsen, J. Z. Uderman, A. Chimiklis, et al., "A randomized clinical trial of Cogmed Working Memory Training in school-age children with ADHD: a replication in a diverse sample using a control condition," Journal of Child Psychology and Psychiatry, vol. 55, pp. 247-255, Mar 2014.

[26] T. Klingberg, E. Fernell, P. J. Olesen, M. Johnson, P. Gustafsson, K. Dahlstrom, et al., "Computerized training of working memory in children with ADHD - A randomized, controlled trial," Journal of the American Academy of Child and Adolescent Psychiatry, vol. 44, pp. 177-186, Feb 2005.

[27] T. Klingberg, H. Forssberg, and H. Westerberg, "Training of working memory in children with ADHD," Journal of Clinical and Experimental Neuropsychology, vol. 24, pp. 781-791, Sep 2002.
[28] A. Al-Shathri, A. Al-Wabil, and Y. Al-Ohali, "Eye-Controlled Games for Behavioral Therapy of Attention Deficit Disorders," in HCI International 2013 - Posters' Extended Abstracts. vol. 373, C. Stephanidis, Ed., ed: Springer Berlin Heidelberg, 2013, pp. 574-578.

[29] NHT. (December 2014) Computer game developed by the Centre to help children with ADHD. Positive magazine, Nottinghamshire Healthcare NHS Trust. 11.

[30] S. Wass, K. Porayska-Pomsta, and M. H. Johnson, "Training Attentional Control in Infancy," Current Biology, vol. 21, pp. 1543-1547, Sep 272011.

[31] N. J. Steiner, E. C. Frenette, K. M. Rene, R. T. Brennan, and E. C. Perrin, "Neurofeedback and Cognitive Attention Training for Children with Attention-Deficit Hyperactivity Disorder in Schools," Journal of Developmental and Behavioral Pediatrics, vol. 35, pp. 18-27, Jan 2014.

[32] C. G. Lim, T. S. Lee, C. T. Guan, D. S. S. Fung, Y. D. Zhao, S. S. W. Teng, et al., "A Brain-Computer Interface Based Attention Training Program for Treating Attention Deficit Hyperactivity Disorder," Plos One, vol. 7, Oct 242012.

[33] U. Strehl, U. Leins, G. Goth, C. Klinger, T. Hinterberger, and N. Birbaumer, "Self-regulation of slow cortical potentials: A new treatment for children with attention-deficit/hyperactivity disorder," Pediatrics, vol. 118, pp. E1530-E1540, Nov 2006.

[34] R. Drechsler, M. Straub, M. Doehnert, H. Heinrich, H. C. Steinhausen, and D. Brandeis, "Controlled evaluation of a neurofeedback training of slow cortical potentials in children with Attention Deficit/Hyperactivity Disorder (ADHD)," Behavioral and Brain Functions, vol. 3, Jul 262007.

[35] M. Arns, S. de Ridder, U. Strehl, M. Breteler, and A. Coenen, "Efficacy of neurofeedback treatment in ADHD: the effects on inattention, impulsivity and hyperactivity: a meta-analysis," Clin EEG Neurosci, vol. 40, pp. 180-9, Jul 2009.

[36] N. Lofthouse, L. E. Arnold, and E. Hurt, "Current status of neurofeedback for attention-deficit/hyperactivity disorder," Curr Psychiatry Rep, vol. 14, pp. 536-42, Oct 2012.

[37] T. Klingberg and F. McNab, "Working memory remediation and the D1 receptor," Am J Psychiatry, vol. 166, pp. 515-6, May 2009.

[38] T. H. van Leeuwen, H. C. Steinhausen, C. C. Overtoom, R. D. Pascual-Marqui, B. van't Klooster, A. Rothenberger, et al., "The continuous performance test revisited with neuroelectric mapping: impaired orienting in children with attention deficits," Behav Brain Res, vol. 94, pp. 97-110, Jul 1998.

[39] J. Swanson, S. Schuck, M. Mann, C. Carlson, K. Hartman, J. Sergeant, et al. (2000, 09/06/2015). Categorical and Dimensional Definitions and Evaluations of Symptoms of ADHD: The SNAP and the SWAN Ratings Scales Available: http://www.adhd.net/SNAP_SWAN.pdf

[40] M. Luman, J. Oosterlaan, and J. A. Sergeant, "The impact of reinforcement contingencies on $\mathrm{AD} / \mathrm{HD}$ : a review and theoretical appraisal," Clin Psychol Rev, vol. 25, pp. 183-213, Feb 2005.

[41] J. P. Zagal and A. Bruckman, "The game ontology project: supporting learning while contributing authentically to game studies," presented at the Proceedings of the 8th international conference on International conference for the learning sciences - Volume 2, Utrecht, The Netherlands, 2008.

[42] J. Carr and P. Blanchfield, "Engaging the Un-Engageable," in Handbook of Research on Improving Learning and Motivation through Educational Games: Multidisciplinary Approaches, P. Felicia, Ed., ed Hershey, PA, USA: IGI Global, 2011, pp. 633657. 